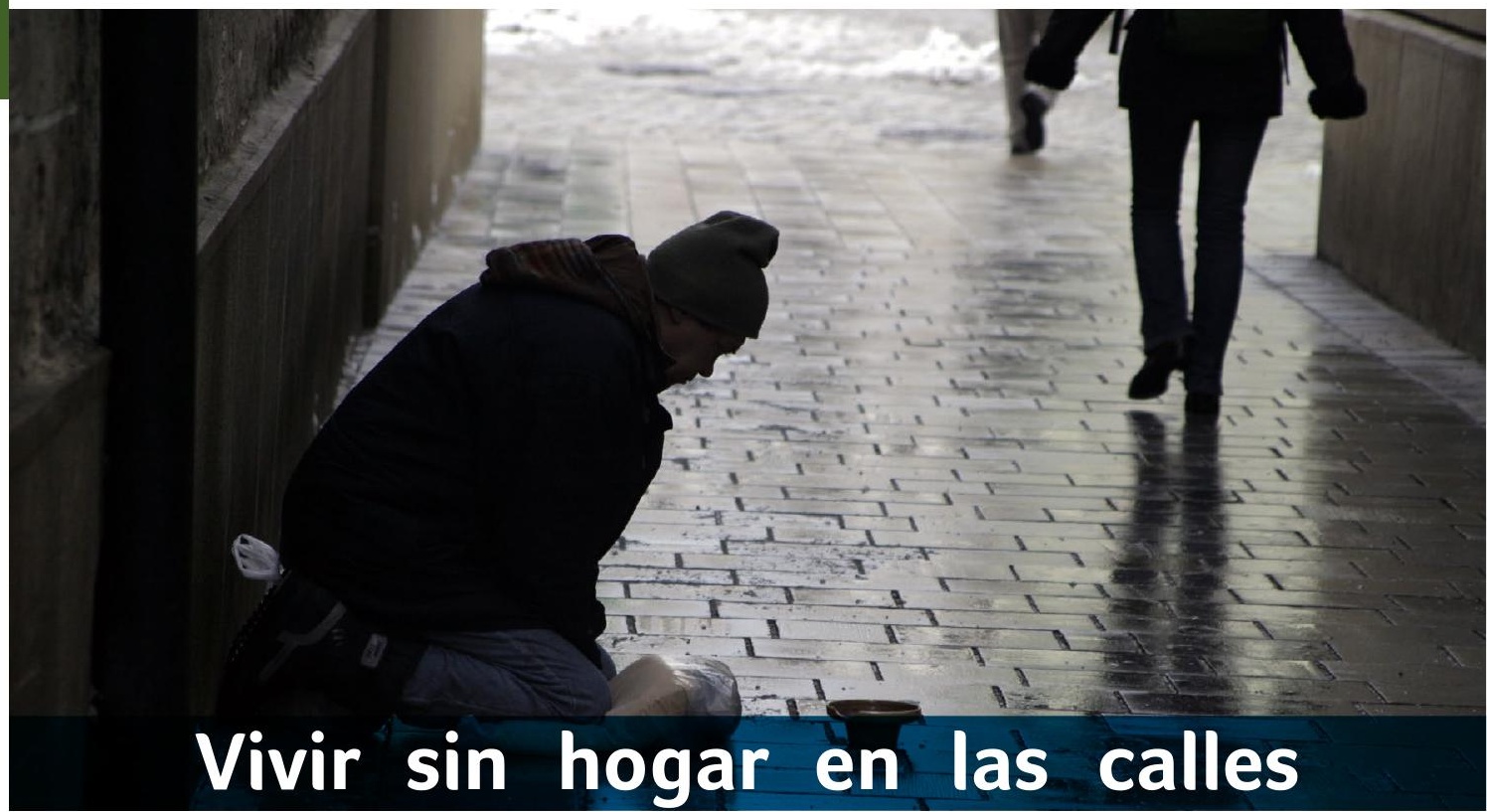

\title{
bogotanas $^{1}$
}

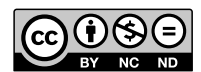

DOI: https://doi.org/10.21158/21451494.v8.n0.2017.2722

Kevin Yesid López-Traslaviña

Corporación Universitaria Minuto de Dios-Uniminuto- klopeztraslauniminuto.edu.co

Edwin Esteban Sánchez-Saiz

Corporación Universitaria Minuto de Dios-Uniminuto - esanchezsaiauniminuto.edu.co

Luis Deiby Cleves-Martínez

Corporación Universitaria Minuto de Dios-Uniminuto - Iclevesmartauniminuto.edu.co

Andrés Felipe Guevara-Fonseca

Corporación Universitaria Minuto de Dios-Uniminuto - aguevarafonauniminuto.edu.co

Cristian David Mejía-Gallego

Corporación Universitaria Minuto de Dios-Uniminuto - cmejiagall2auniminuto.edu.co

1 El profesor James León Parra-Monsalve de la Corporación Universitaria Minuto de Dios-Uniminuto ejercicio como tutor para la elaboración de este texto.

2 Estudiante del programa de Tecnología en Comunicación Gráfica - Corporación Universitaria Minuto de DiosUniminuto. ORCID: https://orcid.org/0000-0002-9934-9477

3 Estudiante del programa de Tecnología en Comunicación Gráfica - Corporación Universitaria Minuto de DiosUniminuto. ORCID: https://orcid.org/0000-0002-5711-6122

4 Estudiante del programa de Tecnología en Comunicación Gráfica - Corporación Universitaria Minuto de DiosUniminuto. ORCID: https://orcid.org/0000-0002-8278-3357

5 Estudiante del programa de Tecnología en Comunicación Gráfica - Corporación Universitaria Minuto de DiosUniminuto. ORCID: https://orcid.org/0000-0002-7129-4857

6 Estudiante del programa de Tecnología en Comunicación Gráfica - Corporación Universitaria Minuto de DiosUniminuto. ORCID: https://orcid.org/0000-0002-5795-3326 
Cómo citar este artículo:

López-Traslaviña, K. Y.; Sánchez-Saiz, E. E.; Cleves-Martínez, L. D.; Guevara-Fonseca, A. F.;

Mejía-Gallego, C. D. (2017). Vivir sin hogar en las calles bogotanas. Revista Comunicación, cultura y política, 8, 50-62.

DOI: https://doi.org/10.21158/21451494.v8.n0.2017.2722

\section{Resumen}

Bogotá alberga alrededor de 9538 personas sin hogar en sus espacios públicos, según el más reciente censo de habitantes de calle. Son estas personas expuestas a todo tipo de violencia y exclusión quienes conforman un grupo altamente vulnerable, en un contexto en el que sus derechos no son reconocidos por el Estado, los grandes medios de comunicación y algunos sectores sociales que los observan con desprecio y prejuicio. En tal sentido, el texto presenta un resumen de la revisión documental que alimenta un proyecto que desarrolla el semillero Miedos Ocultos en la actualidad sobre esta problemática. Este semillero del programa de Tecnología en Comunicación Gráfica de la Corporación Universitaria Minuto de Dios, adelanta actividades de investigación formativa cuyo foco de interés es el área de influencia institucional de la universidad en el sur de Bogotá. Teniendo en cuenta la importancia de la exploración de miradas alternativas frente a un problema persistente como el de las personas que viven en las calles, se establece que la academia, desde la comunicación gráfica en este caso, está llamada a servir desde una perspectiva solidaria e incluyente, que contribuya a eliminar los estigmas y prejuicios que sufre esta población, a través de narrativas innovadoras en el contexto de la realización documental. Así las cosas, se presentan aquí los avances más relevantes de ese proyecto audiovisual, el cual se encuentra en etapa de preproducción.

Palabras clave: Bogotá; habitantes de calle; narrativa documental; inclusión social; proyecto audiovisual; comunicación para el cambio social; narrativa innovadora. 


\title{
Homelessness in the streets of Bogotá
}

\begin{abstract}
According to the most recent census of street dwellers in Bogotá, public spaces in the city shelter about 9538 homeless people. These people are exposed to all kinds of violence and exclusion, and make part of a highly vulnerable group in a context where the State does not recognize their rights, and the large media and some social sectors look upon them with contempt and prejudice. In this sense, this document presents a summary of the documentary review that feeds a project on this issue developed by the seedbed Miedos Ocultos (Hidden Fears). This seedbed from the Technology in Graphic Communication program at Corporación Universitaria Minuto de Dios carries out formative research activities whose focus is the university's area of institutional influence in southern Bogotá. Taking into account the importance of exploring alternative views in the face of a persistent problem such as that of people living on the streets, the study determined that from the graphic communication, the academy is called to serve from a perspective of solidarity and inclusion that contributes to eliminating the stigmas and prejudices suffered by this population, through innovative narratives in documentary filmmaking. Thus, we present the most relevant advances of this audiovisual project, which at this moment is in a preproduction stage.
\end{abstract}

Keywords: Bogotá; street dwellers; documentary narrative; social inclusion; audiovisual project; communication for social change; innovative narrative. 


\section{Viver sem casa nas ruas de Bogotá}

\section{Resumo}

Bogotá abriga, em seus espaços públicos, cerca de 9.538 pessoas sem casa, de acordo com o censo mais recente de moradores de rua. São essas pessoas expostas a todos os tipos de violência e exclusão que constituem um grupo altamente vulnerável, em um contexto em que seus direitos não são reconhecidos pelo Estado, pelos grandes meios de comunicação e por alguns setores sociais que os veem com desprezo e preconceito. Nesse sentido, o texto apresenta um resumo da revisão documental que alimenta um projeto que atualmente desenvolve o grupo de pesquisa Medos Ocultos sobre esse problema. Esse grupo de pesquisa, que faz parte do programa de Tecnologia em Comunicação Gráfica da Corporação Universitária Minuto de Dios, realiza atividades de pesquisa formativa cujo foco de interesse é a área de influência institucional da universidade no sul de Bogotá. Tendo em consideração a importância da exploração de visões alternativas diante de um problema persistente como o das pessoas que moram nas ruas, estabelece-se que a academia, a partir da comunicação gráfica, nesse caso, é chamada a servir de uma forma solidária e inclusiva, a contribuir na eliminação dos estigmas e preconceitos sofridos por essa população, através de narrativas inovadoras no contexto da produção documental. Assim, são aqui apresentados os avanços mais relevantes desse projeto audiovisual, que se encontra em fase de pré-produção.

Palavras-chave: Bogotá; moradores de rua; narrativa documental; inclusão social; projeto audiovisual; comunicação para mudança social; narrativa inovadora. 


\section{Etre sans-abri à Bogotá}

\section{Résumé}

Bogotá abrite selon le dernier recensement des personnes sans domicile fixe, environ 9538 sans-abri dans ses espaces publics. Ces personnes sont exposées à tous types de violence et d'exclusion, et constituent une population extrèmement vulnérable dans la mesure où leurs droits fondamentaux ne sont reconnus ni par l'État, ni par les médias, ni par certains secteurs de la population qui les considérent avec mépris et préjugés. Cet article présentera un résumé documenté, alimentant un projet mis en place par la pépinière Occult Fears qui traite cette problématique. Cette pépinière du programme de technologie en communication graphique de la corporation universitaire Minuto de Dios mène des activités d'investigation formative dans la zone d'influence institutionnelle de l'université, dans la périphérie sud de Bogotá. Dans la mesure où il est essentiel d'explorer des points de vue alternatifs s'agissant d'un phénomène social persistant, le monde universitaire de la communication graphique est appelé à être solidaire et inclusif, et doit contribuer à combattre les stigmates et préjugés subis par cette population au travers de récits innovants dans le cadre de productions documentaires. Nous présenterons ici les avancées les plus pertinentes de ce projet audiovisuel encore en phase de pré-production.

Mots-clés: Bogotá; personnes sans-abri; récit documentaire; inclusion sociale; projet audiovisuel; communication pour le changement social; récit innovant. 


\section{Introducción}

El Centro de Operación San Camilo, adscrito a la Corporación Universitaria Minuto de Dios, se ubica en el sur de Bogotá en la calle $1^{\circ}$ entre las carreras 9 y 10, muy cerca de barrios como San Bernardo, el Parque Tercer Milenio y otras áreas del centro de la ciudad, las cuales han vivido por mucho tiempo la problemática de personas que viven de forma transitoria o permanente en las calles y los sitios públicos. Se trata de un problema cuya solución compete a toda la sociedad y al que nuestra institución educativa puede aportar desde los diversos campos del conocimiento. Por esto, la exploración de miradas alternativas frente a un problema persistente, en el que la comunicación gráfica está llamada a servir desde una perspectiva solidaria e incluyente, contribuye a eliminar estigmas y prejuicios que sufre esta población, a través de narrativas innovadoras en el contexto de la realización documental.

En ese orden de ideas se planteó realizar un documental basado en relatos alternativos e incluyentes de personas con estudios superiores que viven en situación de calle en las localidades bogotanas. El objetivo se fundamenta metodológicamente en la investigación creación, el enfoque cualitativo y cuatro etapas de desarrollo.

La participación en el semillero y la realización de actividades de investigación permitieron el mejor conocimiento del fenómeno y la planificación del documental. Teniendo en cuenta que los medios ocultan mucha información sobre las personas en situación de calle, se encontró una gran diversidad no prevista en principio- entre esta población, lo cual exigió replantear las narrativas propuestas inicialmente. Tales avances se relacionan a continuación, en términos teóricos, metodológicos y de resultados preliminares del proceso investigativo. 


\section{Fundamentación teórica}

La legislación colombiana define a las personas que viven en espacio públicos como «habitantes de la calle» (Ley 1641 de 2013, art. 2), mientras en otros países u organizaciones internacionales las tratan como «personas sin techo» o «sin hogar» (Bainbridge y Carrizales, 2017). Estas nociones evidencian la variedad de formas de definir a la persona que se encuentra en esta situación. Revela, además, la existencia de múltiples factores de carácter personal, económico, social, cultural, político, etc., que motivan la denominación de la gente que hoy vive este drama en muchas ciudades del mundo.

Como se ha señalado, se trata de:

un problema que trasciende las fronteras nacionales, afectando a casi el dos por ciento de la población mundial, principalmente en las grandes aglomeraciones urbanas. Que no está relacionado meramente con las crisis económicas o familiares. Que su comprensión y resolución necesita de la conjunción de esfuerzos del Estado, la academia, los medios de comunicación, la familia y de todos los sectores que puedan aportar desde cada una de sus posibilidades. (Parra-Monsalve, 2020, p. 273)

Las personas sin hogar están entre los grupos más marginalizados de la sociedad. En su mayoría perciben el prejuicio y la discriminación por vivir en la calle, por su aspecto físico o higiénico, su incapacidad mental, color o raza, edad u orientación sexual, en lugares públicos, restaurantes, almacenes, lugares de trabajo, albergues, espacios de recreación y servicios de salud (Rodrigues, Lima y Holanda, 2018; Barata et al., 2015; Bachiller, 2015).

La atención a personas en situación de calle representa un desafío enorme para las administraciones públicas de las grandes ciudades de los países de la OCDE (Bainbridge y Carrizales, 2017). Es una población que, tradicionalmente, se intenta homogeneizar a través de la propia política pública y los estereotipos sociales (Rolnik, 2010). No obstante, enfocarse en sus especificidades y diferencias internas puede ser el mejor camino en la comprensión de sus 
necesidades para la creación de proyectos que contemplen la integralidad en

acciones de salud y servicios sociales que busquen una mayor inclusión social (Barata et al., 2015).

Buena parte del tratamiento que los medios tradicionales dan a estas personas resalta el discurso de la inseguridad y no la privación de derechos a que se ven enfrentadas; de manera que las muestran como parte de un problema a combatir. Al ser clasificadas como «problema» o "peligro» se justifica su exclusión y la naturalización de su situación. Vale decir que los medios regulan lo cotidiano social, esto es, determinan los comportamientos debidos en la sociedad, el orden y el desorden, lo deseable y lo indeseable (Resende, 2012). De este modo, la omisión de la responsabilidad estatal frente a personas con derechos vulnerados es latente en muchos mensajes difundidos por los grandes medios de comunicación. La injusticia social es así naturalizada en textos que refuerzan la exclusión y el estigma sobre grupos vulnerables.

También es importante destacar que existen muchas personas en situación de calle que optan por el anonimato y esto limita un poco más el público estudiado debido a que,

\section{prefieren no ser reconocidos por sus familiares. Además, las cámaras y las grabadoras simbolizan la posibilidad de que la gente los tache como los sin techo, la persona es más que un sin hogar, y para ellos el término entrevista remite a un imaginario basado en la reproducción de estigmas. (Bachiller, 2015)}

Son personas marginadas y estigmatizadas en un contexto que no les permite participar plenamente de la vida ciudadana urbana, en la cual se les violan sus derechos y se generan temores, miedos y angustias que se evidencian en el momento de tratar con quienes viven en esta situación. Personas que temen a los otros, al dolor ocasionado por las heridas, al SIDA, a perder la libertad, a ser «matados», a quedarse en la droga y perder a sus seres queridos (Forero, Giraldo, Valencia, Hurtado y Montoya, 2007). Una población que enfrenta el miedo a través de los significados de miedo-adrenalina y miedo-enemigo: el primero posibilita enfrentar las situaciones, o huir si es necesario, evitando el riesgo para su vida, y el segundo los acobarda y no les permite actuar (Forero et al., 2007). Son seres humanos que experimentan sentimientos de angustia y dolor que aprenden a enfrentar en su cotidiano vivir. 
Reconociendo esas condiciones precarias, la Alcaldía de Bogotá estableció la Política Pública para el Fenómeno de Habitabilidad de Calle mediante el Decreto 560 de 2015. Bajo esta norma se busca,

resignificar el fenómeno de la habitabilidad en calle en Bogotá, por medio de la implementación de acciones estratégicas integrales, diferenciales, territoriales y transectoriales, orientadas al mejoramiento de la convivencia ciudadana y la dignificación de los ciudadanos y ciudadanas habitantes de calle, en el marco de la promoción, protección, restablecimiento y realización de sus derechos, que contribuyan a su inclusión social, económica, política y cultural, así como a la protección integral de las poblaciones en riesgo de habitar la calle (art. 5).

Este es un problema que afecta en especial a las grandes urbes colombianas. Bogotá, por ejemplo, cuenta entre sus habitantes casi diez mil personas en esta situación (DANE, 2017). Además, a pesar del desmantelamiento de «El Cartucho», hace dos décadas, o de «El Bronx», en el 2016, la problemática no parece menguar. Un desafío que implica, como se señaló, el esfuerzo conjunto de todos los sectores sociales, apalancado por políticas de Estado, más allá de las meras intervenciones policivas que se dan de vez en cuando o de periodos de gobierno aislados.

\section{Metodología}

Este es un proyecto de investigación creación que sigue el enfoque cualitativo, aquel que «resulta conveniente para comprender fenómenos desde la perspectiva de quienes los viven y cuando buscamos patrones y diferencias en estas experiencias y su significado» (Hernández y Mendoza, 2018, p. 9). Su alcance es descriptivo, pues se busca «caracterizar un fenómeno, un grupo de personas o un objeto de análisis» (Núñez, Marquina, León y Sánchez, 2017, p. 50). Respecto a las técnicas se eligieron la revisión documental, la observación y la entrevista para la recolección de datos e informaciones sobre y entre el grupo analizado. 
Respecto a la población y la muestra, el universo de personas con estudios superiores en situación de calle en la capital de Colombia es de 405 individuos

(DANE, 2017). Dado que el enfoque propuesto es cualitativo, la muestra se realiza por conveniencia, por lo que serán escogidos tres individuos que se ajusten al perfil específico del estudio, quienes puedan dar su consentimiento para participar en el proyecto como protagonistas de las historias. Se prevé que la selección de la muestra se realice en alguna institución, pública o privada, que brinde apoyo social a estas personas en el centro de la ciudad, dado que el trabajo en las calles puede presentar inconvenientes a la hora de encontrar individuos con dicho perfil.

El proyecto se realiza en cuatro fases: 1) identificación de documentos, búsqueda bibliográfica y cibergráfica; 2) análisis de textos y documentos; 3) trabajo de campo en centros de atención y producción audiovisual; 4) posproducción y publicación del documental.

\section{Análisis de resultados}

El semillero Miedos Ocultos ha indagado temas de investigación en el ámbito de los miedos, fobias, angustias y su relación con la comunicación gráfica. Una de las propuestas que actualmente adelanta trata sobre la habitabilidad de calle en Bogotá. El proyecto busca realizar un documental basado en relatos alternativos e incluyentes de personas con estudios técnicos, tecnológicos, profesionales o de posgrado que se encuentran actualmente en situación de calle en la ciudad.

De acuerdo con lo anterior, se prefirió un enfoque que resalta narrativas sobre miedos, vivencias y sueños de estas personas marginadas por la sociedad y el Estado. Al encontrarse una gran diversidad de grupos que conforman esta población se eligió, de la misma manera, limitar el proceso de indagación a profesionales, tecnólogos y técnicos que viven en la actualidad esta situación, lo cual permite destacar una parte de tal diversidad poblacional. 
Las anteriores reflexiones, así como los debates en los encuentros del semillero, nutrieron el proceso de realización documental y sus dos primeras creaciones. En primer lugar, el guion descriptivo, titulado Una urbe de pesadillas y sueños, permitió visualizar inicialmente la narrativa propuesta, aunque se tuvo en cuenta que por ser un documental se podría transformar. En segundo lugar, el storyboard, realizado a partir del guion descriptivo, se consolidó mediante el trabajo colaborativo de los integrantes del semillero y sirve ahora de base para elaborar el plan de producción audiovisual.

\section{Conclusiones}

Así, entonces, se evidenció, según lo adelantado en el proceso, que son necesarias otras formas de narrar el asunto tratado, debido a la gran diversidad de grupos e individuos que conforman esta población y por ello se eligió limitar el proceso de indagación a profesionales, tecnólogos y técnicos que viven actualmente esta situación, lo cual permite destacar una parte de esa diversidad poblacional, tal como lo recomiendan Barata et al. (2015).

Así mismo, es necesario anotar que con este proyecto de investigación se aporta a la proyección social de la universidad y a consolidar el papel que esta juega en la sociedad. Las experiencias pasadas de las personas sin hogar revelan vivencias desfavorables y situaciones traumáticas, aunque también la conciencia de que están en una situación transitoria. Es un fenómeno que urge comprender mejor desde las instituciones de educación superior. La vulnerabilidad en esta población se manifiesta a través de problemas de diferente índole que no resuelve el Estado ni comprende la sociedad, de modo que se generan miedos y angustias que enfrentan en su cotidianidad.

Se resalta, de igual forma, que la revisión documental es una técnica valiosa para la fundamentación de proyectos de producción audiovisual. Representa un paso indispensable, principalmente en aquellos proyectos que trabajan con poblaciones altamente vulnerables como la de las personas sin hogar. De igual forma, permite afinar el lenguaje y reconocer ángulos insospechados de los fenómenos sociales objeto de producción audiovisual. 
Es necesario señalar, finalmente, cómo la complejidad del fenómeno requiere otros abordajes que complementen su entendimiento. Abordar, por ejemplo, aspectos de orden regional, es decir, de sus características en otras ciudades y territorios colombianos, o de orden mediático, es decir, del tratamiento que los medios, ya sea impresos, radiales, televisivos o electrónicos, dan al problema, mediante el estudio de casos específicos. Estos desarrollos analíticos auxiliarían una mejor comprensión de esta población vulnerable en el futuro.

\section{Referencias}

Bachiller, S. (2015). Reflexiones etnográficas sobre un trabajo de campo con personas en situación de calle. Población \& Sociedad, 22(2), 135-144.

Bainbridge, J.; Carrizales, T. J. (2017). Global homelessness in a post- recession world. Journal of Public Management \& Social Policy, 24(1), 71-90.

Barata, R.; Barradas-Carneiro, J. N.; Sampaio de Almeida, M. C.; Silveira, C. (2015). Desigualdade social em saúde na população em situação de rua na cidade de São Paulo. Saúde e Sociedade, 24(Suppl. 1), 219-232. DOI: https://doi. org/10.1590/s0104-12902015s01019

DANE (Departamento Administrativo Nacional de Estadística). (2017). Censo habitantes de la calle 2017. Bogotá: DANE.

Decreto 560 de 2015. (21 de diciembre de 2015). Por medio del cual se adopta la política pública distrital para el fenómeno de habitabilidad en calle y se derogan los decretos distritales N.os 136 de 2005 y 170 de 2007. Alcaldía Mayor de Bogotá.

Forero, C.; Giraldo, Á.; Valencia, A.; Hurtado, M.; Montoya, B. (2007). Para sobrevivir en la calle hay que tener miedo. Investigación y Educación en Enfermería, 25(2), 28-35.

Hernández, R.; Mendoza, C. (2018). Metodología de la investigación. México: McGraw-Hill Interamericana. DOI: https://doi.org/10.17993/CcyLl.2018.15

Ley 1641 de 2013. (12 de julio de 2013.). Por medio de la cual se establecen los lineamientos para la formulación de la política pública social para habitantes de la calle y se dictan otras disposiciones. Diario Oficial 48849. Congreso de Colombia. 
Núñez, G.; Marquina, O.; León, L.; Sánchez, M. (2017). Guía de investigación en ciencias y artes de la comunicación. Lima: Pontificia Universidad Católica del Perú.

Parra-Monsalve, J. L. (2020). La representación del sinhogarismo en la prensa digital colombiana: la intervención de «el Bronx» (2016) y su cubrimiento en «El Tiempo». Estudios sobre el Mensaje Periodístico, 26(1), 265-274. DOI: https://doi. org/10.5209/esmp.67305

Resende, V. M. (2012). Representação discursiva de pessoas em situação de rua no «Caderno Brasília»: naturalização e expurgo do outro. Ling. (dis)curso, 12(2), 439-465. DOI: https://doi.org/10.1590/S1518-76322012000200004

Rodrigues, J. S.; Lima, A. F.; Holanda, R. B. (2018). Identidade, drogas e saúde mental. Psicologia: Ciência e Profissão, 38(3), 424-436. DOI: https://doi. org/10.1590/1982-37030004912017

Rolnik, R. (20 de diciembre de 2010). Informe de la relatora especial sobre una vivienda adecuada como elemento integrante del derecho a un nivel de vida adecuado y sobre el derecho de no discriminación a este respecto. ONU, Consejo de Derechos Humanos. Recuperado de https://bit.ly/3IZg7fZ 
